PROCEEDINGS OF THE

AMERICAN MATHEMATICAL SOCIETY

Volume 128, Number 8, Pages 2253-2259

S 0002-9939(99)05301-0

Article electronically published on December 7, 1999

\title{
COLLAPSIBILITY OF $\Delta\left(\Pi_{n}\right) / \mathcal{S}_{n}$ AND SOME RELATED CW COMPLEXES
}

\author{
DMITRY N. KOZLOV
}

(Communicated by John R. Stembridge)

\begin{abstract}
Let $\Delta\left(\Pi_{n}\right)$ denote the order complex of the partition lattice. The natural $\mathcal{S}_{n}$-action on the set $[n]$ induces an $\mathcal{S}_{n}$-action on $\Delta\left(\Pi_{n}\right)$. We show that the regular CW complex $\Delta\left(\Pi_{n}\right) / \mathcal{S}_{n}$ is collapsible. Even more, we show that $\Delta\left(\Pi_{n}\right) / \mathcal{S}_{n}$ is collapsible, where $\Pi_{\Delta}$ is a suitable type selection of the partition lattice. This allows us to generalize and reprove in a conceptual way several previous results regarding the multiplicity of the trivial character in the $\mathcal{S}_{n}$-representation on $H_{*}\left(\Delta\left(\Pi_{n}\right)\right)$.
\end{abstract}

\section{INTRODUCTION}

Assume $P$ is a poset equipped with an order-preserving action of a group $G$. This paper grew out of the author's attempts to understand the relation between the topology of $\Delta(P) / G$ and $\Delta(P / G)$, where $\Delta$ denotes the order complex construction and $P / G$ is a category defined as a colimit of a suitable functor (see [4, Definition $2.2])$. In [4 E. Babson and the author have given certain conditions on the action which guarantee that $\Delta(P) / G$ is homeomorphic to $\Delta(P / G)$.

In this paper we are concerned with the case when $P=\Pi_{n}$ or a suitable subposet of it, $G$ is a symmetric group $\mathcal{S}_{n}$, and the action is induced from the $\mathcal{S}_{n}$ action on the ground set $[n]$.

The partition lattice $\Pi_{n}$ is an extensively studied object in algebraic combinatorics. Two important appearances of it are:

- as an intersection lattice of the braid arrangement;

- in studying the complexes of disconnected graphs in connection with Vassiliev knot invariants (see [16, 1]).

It has been proved many times by several means that the order complex of $\Pi_{n}$ is Cohen-Macaulay (see for instance [2] 3]).

It is easy to see that $\Pi_{n} / \mathcal{S}_{n}$ is a category with a terminal object, hence $\Delta\left(\Pi_{n}\right) / \mathcal{S}_{n}$ is contractible. However, since the conditions from [4] are not satisfied in this case we cannot immediately conclude that $\Delta\left(\Pi_{n}\right) / \mathcal{S}_{n}$ is contractible. The purpose of this paper is to show that this is in fact the case. $\Delta\left(\Pi_{n}\right) / \mathcal{S}_{n}$ inherits the cell structure from $\Delta\left(\Pi_{n}\right)$ and we show that, with regard to this cell structure, $\Delta\left(\Pi_{n}\right) / \mathcal{S}_{n}$ is collapsible.

More generally, for any $\Lambda$ - a set of number partitions of $n$-we define $\Pi_{\Lambda}$ to be the subposet of $\Pi_{n}$ consisting of all partitions of the type from $\Lambda$. We show that

Received by the editors August 6, 1998 and, in revised form, September 18, 1998.

2000 Mathematics Subject Classification. Primary 05E25. 
under certain conditions $\Delta\left(\Pi_{n}\right) / \mathcal{S}_{n}$ is collapsible. These conditions can be best understood as follows. Choose $k$ such that $2 \leq k \leq n$ (for example $k=2$ !). Then the conditions of Theorem 4.1 can be reformulated as: if $\lambda \in \Lambda$, then the maximal number partition of the type $\left(k^{m}, 1^{n-k m}\right)$, which refines $\lambda$, is also in $\Lambda$.

When $X$ is a finite simplicial complex and $G$ is a discrete group acting on $X$, it is known that, for any $i$, the Betti number $\beta_{i}(X / G, \mathbf{k})$ is equal to the multiplicity of the trivial character in the induced representation of $G$ on the homology group $H_{i}(X, \mathbf{k})$, where $X / G$ is the quotient space and $\mathbf{k}$ is the field of characteristic 0 or coprime to $|G|$ (see for instance [6, Theorem 1] or [5, page 120, Theorem 2.4]). This allows us in Corollary 4.2 to translate (a weaker version of) our result into the language of the representation theory. Questions of such nature have been studied before, most notably by R. Stanley in [13, P. Hanlon in 10] and S. Sundaram in 14. We give more conceptual proofs for [13] Proposition 7.8], [10, Theorem 3.1] and [14, Theorem 4.2] as the special cases of our result (the authors were working with the case when $\Lambda$ is coming from a rank selection and $k=2$ ). We hope that this sheds some light on the role which the number partitions of the type $\left(k^{m}, 1^{n-k m}\right)$ play in the fact that for certain rank selections the multiplicity of the trivial character in the induced representation on the homotopy groups is 0 .

For the sake of brevity, we have chosen to formulate our argument in terms of Discrete Morse Theory from [8]. In Section 3 we give a simple combinatorial argument for the statement of Discrete Morse Theory that we need (see also [1. Proposition 3.7]).

\section{FRAMEWORK}

Let $\Delta$ denote the nerve functor $\Delta:$ Cat $\rightarrow$ Simplicial Sets, mapping a category $K$ to its nerve $\Delta(K)$ (see [11,12]). In the combinatorial context $\Delta$ has been mostly studied when $K$ is a poset, in which case $\Delta(K)$ has elements of $K$ as vertices and a simplex for each chain (a set of comparable elements). When $K$ is a poset, $\Delta(K)$ is a simplicial complex, which is often called the order complex.

When $X$ is a cell complex, we denote by $P(X)$ its face poset, i.e., the poset whose elements are cells of $X$ and the order relation is inclusion. A triangulated space (cf. [9]) is defined to be a cell complex $X$ such that the lower intervals of $P(X)$ are Boolean algebras. We denote by $|X|$ the geometric realization of $X$. The triangulated spaces make appearance in the topological combinatorics in the following important examples:

- If $K$ is a category, then $\Delta(K)$ is a triangulated space iff $K$ has no inverses. In particular, if $G$ acts on a poset $P$ in an order-preserving way, then $\Delta(P / G)$ is a triangulated space, where $P / G$ is defined as a colimit (cf. [4]).

- If a group $G$ acts on a triangulated space $X$ in such a way that: if a cell $\sigma$ is fixed by $g \in G$, then $\sigma$ is fixed by $g$ pointwise (cf. [5, Condition (A), p. 115]), then $|X| / G$ is homeomorphic to $|X / G|$, where $X / G$ is a certain triangulated space. The cells of $X / G$ are orbits of the cells of $X$ and $p: X \rightarrow X / G$ is a cell map. In particular, if $G$ acts on a poset $P$ in an order-preserving way, then $|\Delta(P)| / G$ is homeomorphic to $|\Delta(P) / G|$ (see [4]). The reader may wish to compare this to the simplicial case (see [5], page 117]).

The language of the elementary collapses will be indispensable for our argument.

Definition 2.1. Let $X$ be a regular CW complex. Assume that $F_{1}$ and $F_{2}$ are cells of $X$ such that $F_{2}$ is a maximal cell which contains $F_{1}$, and there is no other maximal 
cell containing $F_{1}$. A collapse is the replacement of $X$ with $X \backslash\left\{F \mid F_{1} \subseteq F\right\}$. A collapse is called elementary if $\operatorname{dim} F_{1}+1=\operatorname{dim} F_{2}$.

Clearly, a collapse is a strong deformation retract, hence it preserves the homotopy type of the space.

\section{Discrete Morse theory}

Our main tool is the result proved by R. Forman as part of his Discrete Morse theory (see [8]). Although contained in his paper, it is convenient for us to reformulate Forman's result in different notations and give a simple and direct argument.

Let $\Delta$ be a regular CW complex. A matching $W$ on $P=P(\Delta)$ (cf. 8 Definition 9.1]) is a set of disjoint pairs $(\sigma, \tau)$ such that $\tau, \sigma \in P, \tau \succ \sigma$ (" $\succ$ " denotes the covering relation). We set

$$
\begin{aligned}
& \vec{W}=\{\sigma \in P \mid \text { there exists } \tau \text { such that }(\sigma, \tau) \in W\}, \\
& \overleftarrow{W}=\{\tau \in P \mid \text { there exists } \sigma \text { such that }(\sigma, \tau) \in W\} .
\end{aligned}
$$

If $(\sigma, \tau) \in W$, then we set $W(\sigma)=\tau$. If $\sigma \notin \overleftarrow{W} \cup \vec{W}$, then we call the cell $\sigma$ critical. Let $m_{i}(W)$ denote the number of critical $i$-cells.

Definition 3.1 (cf. [8, Definition 9.2]). A matching is called acyclic if it is impossible to find a sequence $\sigma_{0}, \ldots, \sigma_{t} \in \vec{W}$ such that $\sigma_{0} \neq \sigma_{1}, \sigma_{0}=\sigma_{t}$ and $W\left(\sigma_{i}\right) \succ \sigma_{i+1}$ for $0 \leq i \leq t-1$.

The following result is a direct consequence of [8, Theorem 9.3] and [8, Corollary 3.5]. We give a short explicit argument.

Theorem 3.2. Let $\Delta$ be a regular $C W$ complex of dimension $d$, and let $P=P(\Delta)$ be its face poset. Let $W$ be an acyclic matching on $P(\Delta)$.

(1) $\Delta$ is homotopy equivalent to a $C W$ complex $\Delta^{M}$, which has $m_{i}(W)$ cells of dimension $i$.

(2) If the critical cells form a subcomplex $\Delta^{C}$ of $\Delta$, then $\Delta^{C}=\Delta^{M}$ and there is a sequence of elementary collapses leading from $\Delta$ to $\Delta^{M}$.

Proof. A formal proof by induction can be given. However, to underline the simplicity of the situation, we give a direct gluing argument. More precisely, we describe a certain procedure of gluing $\Delta$ from its cells so that the critical cells are glued over their entire boundary and the matched cells are glued in pairs over the entire boundary of their union.

Start with an empty cell complex. At every step, let $x$ be one of the cells of minimal dimension along those which are not yet glued on. Let $\operatorname{dim} x=i$; then the complete $(i-1)$-skeleton is already glued on. If $x$ is critical, then simply glue $x$ to the complex. If $x$ is not critical, then we must have $x \in \vec{W}$. If all of the boundary cells of $W(x)$ (except for $x$ ) are glued on, then just glue $x$ and $W(x)$ to the complex. Otherwise, since the matching $W$ is acyclic, there exists $x_{1}<W(x)$, such that $x_{1} \neq x$ and $x_{1}$ is not yet glued on. Repeat the argument for $x_{1}$. Either we glue on something or, since $W$ is acyclic, we find $x_{2} \neq x, x_{2} \neq x_{1}, W\left(x_{1}\right)>x_{2}$ such that $x_{2}$ is not yet glued, etc.

Since there are only finitely many cells, the process will terminate and some gluing will occur. Since gluing on a pair of two matched cells over the entire boundary does not change the homotopy type (the inverse of such gluing is an elementary collapse) we prove (1). 
To prove (2) simply observe that if the critical cells form a subcomplex, then we can glue them all on in the beginning of the process described above.

\section{RANK SELECTIONS OF THE PARTition LATtiCE}

Let $\Pi_{n}$ be the partition lattice with the ground set $[n]$. Let $\Lambda$ be a set of some number partitions of $n$, such that $(1, \ldots, 1) \notin \Lambda,(n) \notin \Lambda$. Denote by $\Pi_{\Lambda}$ the subposet of $\Pi_{n}$ consisting of all partitions of type from $\Lambda$. In Figure 11 we have pictured the triangulated space $\Delta\left(\bar{\Pi}_{5}\right) / \mathcal{S}_{5}$, where $\bar{\Pi}_{n}$ is $\Pi_{n}$ with maximal and minimal elements removed. It consists of five triangles, four in the background, and the fifth is filled with diagonal lines. It is readily seen that $\left(\bar{\Pi}_{5}\right) / \mathcal{S}_{5}$ is collapsible.

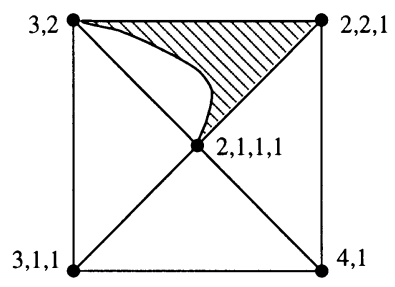

FigURE 1.

When $\lambda$ is a number partition of $n, \lambda=\left(\lambda_{1}, \ldots, \lambda_{t}\right)$, we define $s_{k}(\lambda)=\sum_{i=1}^{t}\left\lfloor\frac{\lambda_{i}}{k}\right\rfloor$ and $\mu_{k}(\lambda)=\left(k^{s_{k}(\lambda)}, 1^{n-k \cdot s_{k}(\lambda)}\right)$. Clearly $\mu_{k}(\lambda)$ refines $\lambda$. The next theorem is the main result of this paper. It gives a condition on $\Lambda$ which guarantees that $\Delta\left(\Pi_{\Lambda}\right) / \mathcal{S}_{n}$ is collapsible.

Theorem 4.1. Let $n>k \geq 2$. Assume that for each partition $\lambda \in \Lambda$ we have $\mu_{k}(\lambda) \in \Lambda$. Then $X_{\Lambda}=\Delta\left(\Pi_{\Lambda}\right) / \mathcal{S}_{n}$ is collapsible.

Proof. As mentioned before, $X_{\Lambda}$ is a triangulated space and $p: \Delta\left(\Pi_{\Lambda}\right) \rightarrow X_{\Lambda}$ is a cell map. The vertices of $X_{\Lambda}$ are indexed by the set $\Lambda$. Elements of $\Lambda$ are partially ordered by refinement and this induces an order on the vertices of each simplex of $X_{\Lambda}$. We call the vertices which have the form $\left(k^{1}, 1^{n-k a}\right)$, for some $a$, special.

Let us now describe an acyclic matching on $P=P\left(X_{\Lambda}\right)$. Let $F \in P$. If all vertices of $F$ are special, then we put $F$ in the set of critical simplices. If not, define $\lambda(F)$ to be the smallest not special vertex of $F$. By the assumption of the theorem we have $\mu_{k}(\lambda) \in \Lambda$. If $\mu_{k}(\lambda)$ is a vertex of $F$, then we put $F$ in $\overleftarrow{W}$, otherwise we put $F$ in $\vec{W}$. This defines a partition of simplices of $X_{\Lambda}$ into three sets: the critical simplices, $\overleftarrow{W}$, and $\vec{W}$.

Claim. For each $F \in \vec{W}$, there exists a unique $\widetilde{F} \in \overleftarrow{W}$ such that $\widetilde{F}$ covers $F$, and $\mu_{k}(\lambda(F))$ is a vertex of $\widetilde{F}$.

Proof. For brevity, we write $\lambda$, resp. $\mu$, instead of $\lambda(F)$, resp. $\mu_{k}(\lambda(F))$, in the rest of the proof.

Existence. Let $H$ be a simplex of $\Delta\left(\Pi_{\Lambda}\right)$ such that $p(H)=F$. Then $H$ is a chain in $\Pi_{\Lambda}$ of the form $\left(a_{1}, \ldots, a_{t}, b, c_{1}, \ldots, c_{q}\right)$, where $a_{i}$ is a set partition of the type $\left(k^{n_{i}}, 1^{n-k n_{i}}\right), b$ has type $\lambda$, and $n_{t}<s_{k}(\lambda)$. By an obvious combinatorial argument there exists a chain $\widetilde{H}=\left(a_{1}, \ldots, a_{t}, a, b, c_{1}, \ldots, c_{q}\right)$ such that $a$ has type $\mu$. Clearly, $\widetilde{F}=p(\widetilde{H})$ belongs to $\overleftarrow{W}$, it covers $F$, and $\mu$ is a vertex of $\widetilde{F}$. 
Uniqueness. Assume that $\widetilde{F}, \widetilde{F}^{\prime} \in \overleftarrow{W}$ both cover $F$, and $\mu$ is a vertex of both $\widetilde{F}$ and $\widetilde{F}^{\prime}$. Choose $\widetilde{H}, \widetilde{H}^{\prime} \in \Delta\left(\Pi_{\Lambda}\right)$ such that $p(\widetilde{H})=\widetilde{F}$ and $p\left(\widetilde{H}^{\prime}\right)=\widetilde{F}^{\prime}$. Because of the conditions on $\widetilde{F}$ and $\widetilde{F}^{\prime}$ and the definition of $p$ we can choose $\widetilde{H}$ and $\widetilde{H}^{\prime}$ so that $\widetilde{H}=\left(a_{1}, \ldots, a_{t}, a, b, c_{1}, \ldots, c_{q}\right), \widetilde{H}^{\prime}=\left(a_{1}, \ldots, a_{t}, a^{\prime}, b, c_{1}, \ldots, c_{q}\right)$, where, as before, $a_{i}$ has type $\left(k^{n_{i}}, 1^{n-k n_{i}}\right), a, a^{\prime}$ have the type $\mu$ and $b$ has type $\lambda$.

$a^{\prime}$ is constructed by cutting each block of $b$ into at most $k-1$ singletons and some blocks of size $k$. Since there is essentially a unique way to refine $b$ in this way, we can replace $\widetilde{H}^{\prime}$ with $g \widetilde{H}^{\prime}$ for $g \in \mathcal{S}_{n}$ such that

- $g a^{\prime}=a$;

- each block of the set partition $b$ is fixed;

- each block of size $k$ of $a_{t}$ is fixed.

Then the chain $\left(a_{1}, \ldots, a_{t}, b, c_{1}, \ldots, c_{q}\right)$ is fixed and hence $g \widetilde{H}^{\prime}=\widetilde{H}$. This proves that $p(\widetilde{H})=p\left(\widetilde{H}^{\prime}\right)$, hence $\widetilde{F}=\widetilde{F}^{\prime}$.

For $F \in \vec{W}$ define $W(F)=\widetilde{F}$, where $\widetilde{F}$ is as in the claim above. Since $F$ is obtained from $\widetilde{F}$ by removing a vertex, we conclude that $W$ is a bijection.

Assume there exists a sequence $\sigma_{0}, \ldots, \sigma_{t} \in \vec{W}$ such that $\sigma_{0} \neq \sigma_{1}, \sigma_{0}=\sigma_{t}$ and $W\left(\sigma_{i}\right) \succ \sigma_{i+1}$ for $0 \leq i \leq t-1$. Assume $W\left(\sigma_{0}\right)=\left(a_{1}, \ldots, a_{\alpha}, b_{1}, \ldots, b_{\beta}\right)$, where $a_{i}$ 's are special and $b_{1}$ is not. Then $\sigma_{0}=\left(a_{1}, \ldots, a_{\alpha-1}, b_{1}, \ldots, b_{\beta}\right)$. Since $\sigma_{1} \in \vec{W}$, and $W\left(\sigma_{0}\right) \neq W\left(\sigma_{1}\right)$, we have $\sigma_{1}=\left(a_{1}, \ldots, a_{\alpha}, b_{2}, \ldots, b_{\beta}\right)$. We see that the number of special vertices in $\sigma_{1}$ is larger by 1 than in $\sigma_{0}$. Repeating the argument, we see that $\sigma_{t}$ has $t$ special vertices more than $\sigma_{0}$, therefore $\sigma_{0} \neq \sigma_{t}$. This leads to the conclusion that $W$ is an acyclic matching.

The critical simplices form a subcomplex of $X_{\Lambda}$, which we call $X_{\Lambda}^{C}$. By Theorem 3.2 there is a sequence of elementary collapses from $X_{\Lambda}$ to $X_{\Lambda}^{C}$. Observe that if $A=\left(a_{1}, \ldots, a_{t}\right)$ and $B=\left(b_{1}, \ldots, b_{t}\right)$ are two simplices of $\Delta\left(\Pi_{\Lambda}\right)$ such that for $i=1, \ldots, t$, both $a_{i}$ and $b_{i}$ are of the type $\left(k^{\alpha_{i}}, 1^{n-k \alpha_{i}}\right)$ for some $\alpha_{i}$, then there exists $g \in \mathcal{S}_{n}$ such that $g A=B$, i.e., $p(A)=p(B)$. This implies that $X_{\Lambda}^{C}$ is a simplex, so we can conclude that $X_{\Lambda}$ is collapsible.

Let $\mathbf{k}$ be a field such that either char $\mathbf{k}=0$ or char $\mathbf{k}>n$. Following a conjecture of R. Stanley [13, page 151], P. Hanlon has proved in [10. Theorem 3.1] that if $\Pi_{n}^{t}$ is the $\{1, \ldots, t\}$ rank selection of the partition lattice, then the multiplicity of the trivial character in the representation $\mathcal{S}_{n} \rightarrow G L\left(H_{i}\left(\Delta\left(\Pi_{n}^{t}\right), \mathbf{k}\right)\right)$ is 0 for all $i$ and $t$. The following corollary generalizes his result.

Corollary 4.2. Assume $\Lambda$ is as in Theorem 4.1. Then the multiplicity of the trivial character in the representation $\mathcal{S}_{n} \rightarrow G L\left(H_{i}\left(\Delta\left(\Pi_{\Lambda}\right), \mathbf{k}\right)\right)$ is 0 for all $i$.

Proof. Again let $X_{\Lambda}=\Delta\left(\Pi_{\Lambda}\right) / \mathcal{S}_{n}$. By [6, Theorem 1], $\beta_{i}\left(X_{\Lambda}, \mathbf{k}\right)$ is equal to the multiplicity of the trivial character in $\mathcal{S}_{n} \rightarrow G L\left(H_{i}\left(\Delta\left(\Pi_{\Lambda}\right), \mathbf{k}\right)\right)$. Thus the statement of the corollary is equivalent to saying that $X_{\Lambda}$ is $\mathbf{k}$-acyclic, which is immediate from Theorem 4.1.

When $\lambda$ is a number partition of $n, \Pi_{\lambda}$ denotes the subposet of $\Pi_{n}$ consisting of all elements which are joins of elements of type $\lambda$. One can view $\Pi_{\lambda}$ as an intersection lattice of the corresponding subspace arrangement. A special interesting case of Corollary 4.2 which is new is a $\{1, \ldots, t\}$ rank selection of $\Pi_{\lambda}$ for $\lambda=\left(k^{m}, 1^{n-k m}\right)$ for some $k$ and $m$. 
It also follows from Theorem 4.1 that if $\Lambda$ is a rank selection such that the ranks $1, \ldots,\left\lfloor\frac{n}{2}\right\rfloor$ are among the selected ones, then the multiplicity of the trivial character as above is 0 . Up to a few border cases, this is [14, Theorem 4.2]. We refer the interested reader to [14] for a thorough account of known results and open questions about the multiplicity of the trivial character in the representation of $\mathcal{S}_{n}$ on the homology groups of rank selections of the partition lattice, as well as for an extensive amount of computational data.

In general, in our terminology, all the questions and results of this kind can be reformulated as studying the Betti numbers of the subcomplexes of one large complex $\Delta\left(\Pi_{n}\right) / \mathcal{S}_{n}$, which are obtained by deleting a certain set of vertices (and their open stars). As we have seen, sometimes this point of view may lead to a more structural understanding of the situation.

We finish this section with yet another application of Theorem 4.1. Let $\Delta_{n}$ denote the simplicial complex whose $k$-faces are all disconnected graphs on $n$ vertices having $k$ edges and the face structure of $\Delta_{n}$ is induced by the inclusions of the graphs. These complexes have been introduced and studied by V. Vassiliev in [16 in connection with his construction of knot invariants. It follows from 15, Theorem 1] that $\Delta_{n}$ is $\mathcal{S}_{n}$-homotopy equivalent to $\Delta\left(\Pi_{n}\right)$ (cf. [16], [1, Proposition 2.1(i)]). This implies that $\Delta_{n} / \mathcal{S}_{n} / \simeq \Delta\left(\Pi_{n}\right) / \mathcal{S}_{n}$. Therefore by Theorem 4.1 we have

Corollary 4.3. $\Delta_{n} / \mathcal{S}_{n}$ is contractible.

\section{ACKNOWLEDGMENTS}

I am indebted to Anders Björner, Eva-Maria Feichtner and Eric Babson for the stimulating conversations at different points of my contemplations on the subject. I would also like to thank the anonymous referee for the valuable comments in improving the first version of this paper.

\section{REFERENCES}

[1] E. Babson, A. Björner, S. Linusson, J. Shareshian, V. Welker, Complexes of not i-connected graphs, Topology 38 (1999), 271-299. CMP 99:05

[2] A. Björner, Shellable and Cohen-Macaulay partially ordered sets, Trans. Amer. Math. Soc. 260 (1980), 159-183. MR 81i:06001

[3] A. Björner, Subspace arrangements, in "First European Congress of Mathematics, Paris 1992" (eds. A. Joseph et. al.), Progress in Math. 119, Birkhäuser, 1994, pp. 321-370. MR 96h:52012

[4] E. Babson, D. N. Kozlov, Group actions on posets, preprint, 1998.

[5] G. E. Bredon, Introduction to compact transformation groups, Academic Press, 1972.

[6] P. E. Conner, Concerning the action of a finite group, Proc. Nat. Acad. Sci. U.S.A. 42 (1956), 349-351. MR 18:61a

[7] E. M. Feichtner and D. N. Kozlov, On subspace arrangements of type D, preprint 489/1995, TU Berlin, 21 pages, (to appear in the special FPSAC'96 issue of Discr. Math.).

[8] R. Forman, Morse theory for cell complexes, Adv. Math. 134 (1998), no. 1, 90-145. MR 99b:57070

[9] S. Gelfand and Y. Manin, Methods of homological algebra, Translated from the 1998 Russian original, Springer, Berlin, 1996. MR 97j:18001

[10] P. Hanlon, A proof of a conjecture of Stanley concerning partitions of a set, European J. Combin. 4 (1983), no. 2, 137-141. MR 85b:05020

[11] D. Quillen, Higher algebraic K-theory I, Lecture Notes in Mathematics, vol. 341, SpringerVerlag, 1973, pp. 85-148. MR 49:2895

[12] G. Segal, Classifying spaces and spectral sequences, Inst. Hautes Études Sci. Publ. Math. No. 34, 1968, pp. 105-112. MR 38:718

[13] R. P. Stanley, Some aspects of groups acting on finite posets, J. Combin. Theory Ser. A 32 (1982), no. 2, pp. 132-161. MR 83d:06002 
[14] S. Sundaram, The homology representations of the symmetric group on Cohen-Macaulay subposets of the partition lattice, Adv. in Math. 104 (1994), 225-296. MR 96c:05189]

[15] J. Thévenaz, P. J. Webb, Homotopy equivalence of posets with a group action, J. Comb. Theory, Ser. A 56 (1991), no. 2, 173-181. MR 92k:20049

[16] V. A. Vassiliev, Complexes of connected graphs, in: L. Corwin et al. (eds.), The Gelfand Mathematical Seminar, 1990-1992, Birkhäuser, Boston, pp. 223-235. MR 94h:55032

Institute for Advanced Study, Olden Lane, Princeton, New Jersey 08540

E-mail address: kozlov@math.ias.edu

Current address: Department of Mathematics, Royal Institute of Technology, 10044 Stockholm, Sweden 